\title{
BMJ Open Teaching strategies and outcome assessments targeting critical thinking in bachelor nursing students: a scoping review protocol
}

\author{
Frida Westerdahl (D , ${ }^{1}$ Elisabeth Carlson, ${ }^{1}$ Anne Wennick, ${ }^{1}$ Gunilla Borglin ${ }^{1,2}$
}

To cite: Westerdahl F, Carlson E, Wennick A, et al. Teaching strategies and outcome assessments targeting critical thinking in bachelor nursing students: a scoping review protocol. BMJ Open 2020;10:e033214. doi:10.1136/ bmjopen-2019-033214

- Prepublication history and additional material for this paper are available online. To view these files, please visit the journal online (http://dx.doi. org/10.1136/bmjopen-2019033214).

Received 25 July 2019 Revised 01 November 2019 Accepted 13 January 2020

\section{Check for updates}

(c) Author(s) (or their employer(s)) 2020. Re-use permitted under CC BY-NC. No commercial re-use. See rights and permissions. Published by BMJ.

${ }^{1}$ Department of Care Science, Malmö University, Malmö,

Sweden

${ }^{2}$ Nursing Education, Lovisenberg Diaconal University College, Oslo, Norway

Correspondence to

Frida Westerdahl;

frida.nygren@mau.se

\section{ABSTRACT}

Introduction Applying critical thinking is essential for nursing students both in an academic and clinical context. Particularly, as critical thinking is a vital part of nurses' everyday problem-solving and decision-making processes. Therefore, regardless of the topic taught or the setting in which it is taught, it requires teaching strategies especially targeting students' critical thinking skills and abilities. One challenge with the latter is the difficulties to assess and evaluate the impact of such teaching strategies on the students' critical thinking disposition. Hence, our objective will be to review published literature on; existing teaching strategies and outcomes assessments targeting nursing students' critical thinking skills and abilities.

Methods and analysis Our scoping review will be conducted in accordance with Arksey and 0'Malley's framework for scoping studies. Search strategies will be developed in cooperation with an experienced librarian, and adjusted to each individual database for example, CINAHL, PubMed, PsycINFO, ERIC and ERC. A preliminary search in CINAHL was conducted on the $17^{\text {th }}$ of July 2019. Peer-reviewed published studies conducted with a qualitative, quantitative or mixed method design and focussing our objectives, will be eligible for inclusion. Included studies will be quality assessed in accordance with their study design. Data will be charted using a standardised extraction form. The qualitative data will be presented through a thematic analyses, and the quantitative data by descriptive numerical analysis. Lastly, nurse educators and nursing students will be consulted for validation of the findings from the scoping review.

Ethics and dissemination Under the Swedish Ethical Review Act (2003:460) this study does not need ethical clearance by a Regional Ethical Review Authority as it not includes any primary empirical data on biological material or sensitive information. The findings will be used to inform the design of a future study aiming to develop an, and subsequently evaluate it, educational intervention targeting teaching strategies focussing on nursing students' critical thinking skills and abilities.

\section{INTRODUCTION}

Applying critical thinking is essential for bachelor nursing students (hereafter nursing students); particularly, considering the complex care situations they regularly will find
Strengths and limitations of this study

To ensure rigour and transparency the upcoming scoping review will be based on (1) a solid methodological framework for scoping studies and (2) the Preferred Reporting Items for Systematic reviews and Meta-Analyses extension for Scoping Reviews checklist.

- A minimum of two members of the review team will independently assess study eligibility.

- Eligible studies will be quality assessed in accordance with their study design.

- To achieve a comprehensive picture of the existing research qualitative, quantitative and mixed methods designs will be included in this scoping review.

- One limitation might be the potential risk for publication bias since grey literature will not be included, as this will facilitate charting of teaching strategies and outcome assessments targeting critical thinking skills and abilities as described solely in published research.

themselves in after graduation. ${ }^{1}$ Care situations that among others require them to work in accordance with established standards ${ }^{2}$ to be able to contribute to a safe, evidence based and optimal clinical practice. Given that nursing is based on scientific knowledge, critical thinking is the reasonable reflection to justify nursing actions based on evidence. Skills and abilities in critical thinking have consequently been found to predict nursing competence together with working years, position, title and educational level, that is, Bachelor or Master in Nursing. ${ }^{3}$ Critical thinking is, therefore, a crucial component of every registered nurse's daily activities, aiding problem-solving and decision-making processes. ${ }^{4}$

According to Scheffer and Rubenfeld the ability to execute critical thinking in nursing could be seen from two perspectives; habits of the mind (cognition), and skills employed by the critical thinker. ${ }^{5}$ Critical thinking can also 
be seen as a consecutive process including (i) gathering information, (ii) questioning, (iii) analysis and evaluation and (iv) problem-solving and application of theory, that is, the nursing process. ${ }^{6}$ This consecutive process of critical thinking needs to be applied both in the clinical area and in the classroom. ${ }^{7}$ However, to develop this ability among nursing students is a complex process. To apply critical thinking, the necessary skills and abilities need to be taught and developed during both the students' clinical placements as well as during their theory courses throughout the nursing education. ${ }^{4}$

One challenge with the concept of critical thinking, often highlighted in the literature, and despite its priority within the nursing education, is the interchangeable use of the concepts of critical thinking, clinical reasoning and clinical judgement. ${ }^{7-9}$ Concepts that Victor-Chmil describe as; 'they are not one and the same' ( $p$ 34). It needs to be acknowledged, as the authors of this current protocol do, that critical thinking often is used as a broader term which includes the concepts of clinical reasoning and clinical judgement. ${ }^{8}$ According to Alfaro-LeFevre clinical reasoning refers to the process used to solve clinical issues and clinical judgement refers to the outcome or conclusion of this process. ${ }^{7}$ Therefore, regardless of the topic taught or the setting in which it is taught, requires teaching strategies especially targeting nursing students' critical thinking skills and abilities. For these strategies to be favourable, it requires implementation throughout the nursing education, and thereby reflected in all parts of the nursing programmes' learning objectives and curricula. ${ }^{10}$ It has been outlined that teaching strategies such as, problem-based learning, concept-mapping, case-based learning interventions and reflective writing are often used in nursing programmes to support critical thinking. ${ }^{6}{ }^{10} 11$ However, another challenge with critical thinking, besides the interchangeable use of concepts, is the difficulty to assess and evaluate the impact of different teaching strategies on the students' critical thinking disposition (ie, skills and abilities) as well as the assessment of the different components in the critical thinking process. ${ }^{8}$ Previous reviews in the current research area have only included either experimental studies ${ }^{12}$ or randomised clinical trials ${ }^{13}$ measuring the effectiveness of teaching strategies. Further, other reviews have involved mixed populations including not only nursing students, but also working nurses and nursing managers ${ }^{14}$ and midwifery students. ${ }^{15}$ Since critical thinking is a vital part of registered nurses' problem-solving and decision-making, this ability needs to be taught already during the nursing education. It is therefore necessary to focus the educational context of undergraduate nursing taking an extended approach on how teaching strategies targeting critical thinking are described, experienced and assessed. Hence, our overarching objective will be to review published literature on; existing teaching strategies and outcomes assessments targeting nursing students' critical thinking skills and abilities.

\section{METHODS AND ANALYSIS}

The upcoming scoping review will address a broad topic (ie, teaching strategies targeting nursing students' critical thinking skills and abilities, as well as outcome assessments of such skills and abilities), where a diverse range of study designs can be considered relevant in answering our additionally wide review questions. Our scoping review will therefore be designed in accordance with Arksey and O'Malley's methodological framework for scoping studies. ${ }^{16}$ However, our design will also be informed by other more recent methodological accounts. ${ }^{17} 18$ The framework will enable us to identify existing gaps in the literature as well as to summarise, evaluate and disseminate the overall state of research activities within the field. ${ }^{16}$ The Preferred Reporting Items for Systematic reviews and Meta-Analyses extension for Scoping Reviews checklist (PRISMA-ScR) was used to prepare this protocol. ${ }^{19}$ PRISMA-ScR will also form the base for the upcoming scoping review as standardised reporting guidelines can according to Colquhoun et al support the critical appraisals of published reviews by expanding on their transparency and reproducibility. ${ }^{20}$

\section{Stage 1: identifying the research question}

The research questions for the upcoming scoping review aims for comprehensiveness, that is, they will be broad to cover the breadth of research evidence in our field of focus. As scoping is an iterative methodological process, ${ }^{16}$ it is possible for us to decide to add supplementary questions based on the findings emerging during the review process. A modified ${ }^{21}$ PICOS (Population, Intervention, Comparison, Outcome and Study Setting) framework will aid us in determining the appropriateness of the research questions, as well as guide us in our database searches (table 1).

\begin{tabular}{|c|c|}
\hline Criteria & Determinants \\
\hline Population & $\begin{array}{l}\text { Nursing students and/or nurse educators at } \\
\text { any semester of study/educational year }\end{array}$ \\
\hline Intervention & $\begin{array}{l}\text { Teaching strategies } \\
\text { Educational strategies } \\
\text { Learning strategies }\end{array}$ \\
\hline Comparison & $N A$ \\
\hline Outcome & $\begin{array}{l}\text { Critical thinking, and/or critical thinking } \\
\text { skills, abilities, dispositions } \\
\text { Outcome assessments for critical thinking, } \\
\text { and/or critical thinking skills, abilities or } \\
\text { disposition }\end{array}$ \\
\hline Study setting & $\begin{array}{l}\text { Nursing programmes leading to a Bachelor } \\
\text { of Nursing offered worldwide at any higher } \\
\text { educational institutions } \\
\text { Academic context (ie, during theoretical } \\
\text { studies) and clinical context (ie, during } \\
\text { clinical placement) }\end{array}$ \\
\hline
\end{tabular}


Since the subsequent goal of the upcoming scoping review is to further the knowledge and understanding about how nurse educators via teaching strategies can target the development of nursing students' critical thinking skills and abilities we will additionally engage in findings of relevance to this. The following tentative research questions were developed to capture the objectives of the upcoming study:

- Which are the teaching strategies described in the literature as targeting critical thinking skills and abilities among nursing students?

- How are these teaching strategies conceptualised, described and experienced by students and/or nurse educators for example, pros and cons?

- Which outcomes are described in the literature as used to assess critical thinking skills and abilities?

\section{Stage 2: identifying relevant studies}

The upcoming scoping review will include primary studies utilising qualitative, quantitative and mixed methods, published in peer-reviewed journals. This strategy will support us to achieve a comprehensive picture of the existing research focussing peer-reviewed studies on teaching strategies targeting critical thinking skills and abilities among nursing students, as well as on existing research focussing on outcome assessments of such skills and abilities. No limits will be applied concerning publication year, since we aim at conducting a comprehensive overview of published studies. Studies will be excluded if the population is not identifiable, qualitative and quantitative data is not possible to extract in case of mixed method design or published in other languages than English. All reasons for exclusion will be documented.

In our upcoming scoping review the term 'teaching strategies' will be used. Thus, our focus is not the overall educational organisation of teaching (ie, educational strategies) or the students' individual general learning process (ie, learning strategies). However, as we are aware of the commonly interchangeable use in the literature of the terms; teaching strategies, educational strategies and learning strategies, they will all be included in our searches. Here the term teaching strategies are operationalised in accordance with Banning, and as encompassing three different perspectives; (i) the didactic perspective, which is teacher centred and mainly involves lectures; (ii) the facilitative perspective, focussing on self-directed learning making the students articulate their knowledge and lastly (iii) the Socratic perspective which is emphasising student-centredness and use objective questioning from the teacher. ${ }^{22}$

The following databases; CINAHL, PubMed, PsycInfo, ERIC and ERC will be used to search for eligible studies. These databases are chosen to cover a comprehensive sample of literature from healthcare science and education. A search strategy for each database will be developed by the review team with assistance from an experienced librarian. Our strategies will include both database specific heading that is, Medical Subject
Headings, keywords and synonyms. All specific headings and key words will be combined using the Boolean operators OR as well as AND. To ensure comprehensiveness, included studies reference lists will be manually searched. As outlined by Arksey and O'Malley the search strategy should be an iterative process and the search terms could be adjusted while an increased familiarity with the literature is achieved. For this reason, a preliminary pilot search strategy will be applied to the databases and the first 100 search results will be reviewed by the review team to assess validity. ${ }^{16}$ During the review team meetings, adjustments will be applied to the search strategy and search terms until full agreement is reached. Grey literature (ie, literature that is not formally published in sources such as journal articles or books) will not, as described elsewhere, be included in our upcoming scoping study. ${ }^{23}$ This will support us to focus on and to chart how teaching strategies targeting skills and abilities such as critical thinking is described in published peer-reviewed research. A draft of a preliminary search in CINAHL conducted on the $17^{\text {th }}$ of July 2019 is attached in online supplementary file 1.

\section{Stage 3: study selection}

The study selection will first consist of a title and abstract scan. If the title and abstract are in line with the scoping review's objectives and questions to the literature or if the relevance of the study is unclear a full-text review will follow. Retrieved studies from each database are going to be divided equally among a minimum of two reviewers, who independently will conduct the selection process. ${ }^{17}$ To facilitate the process, we are going to use the data programme Rayyan. The programme is a mobile and web application developed to facilitate the screening of title and/or abstract as well as the collaboration between the reviewers. ${ }^{24}$ During the study selection process, the first reviewer (FW) will be responsible for regularly convoking the review team for discussions concerning uncertainties and to refine the study selection process. ${ }^{17}$ Criteria for inclusion can also be applied ad hoc during the process when acquaintance with the field of research is increased. ${ }^{16}$ If any disagreements on study inclusion occur, an additional reviewer will be consulted to determine the final inclusion. ${ }^{17}$ The study selection process (figure 1) will be accounted for by the PRISMA flow diagram. ${ }^{25}$

Contrary to Arksey and O'Malley's methodological framework, ${ }^{16}$ studies eligible for inclusion in our scoping review are going to be quality assessed. The assessment of the included studies' quality will allow us to identify where the research itself is of poor quality, that is, identifying gaps in the existing literature review. According to Grant and Booth the lack of quality assessments in scoping reviews are likely to limit the uptake of the findings. ${ }^{26}$ Their sentiment is supported by both Levac, Colquhoun and O'Brien ${ }^{17}$ and Daudt, van Mossel and Scott ${ }^{18}$ who state that a quality assessment of included studies will likely result in findings more useful for practice. The quality assessment will be conducted by a minimum of two reviewers, who will use the relevant 


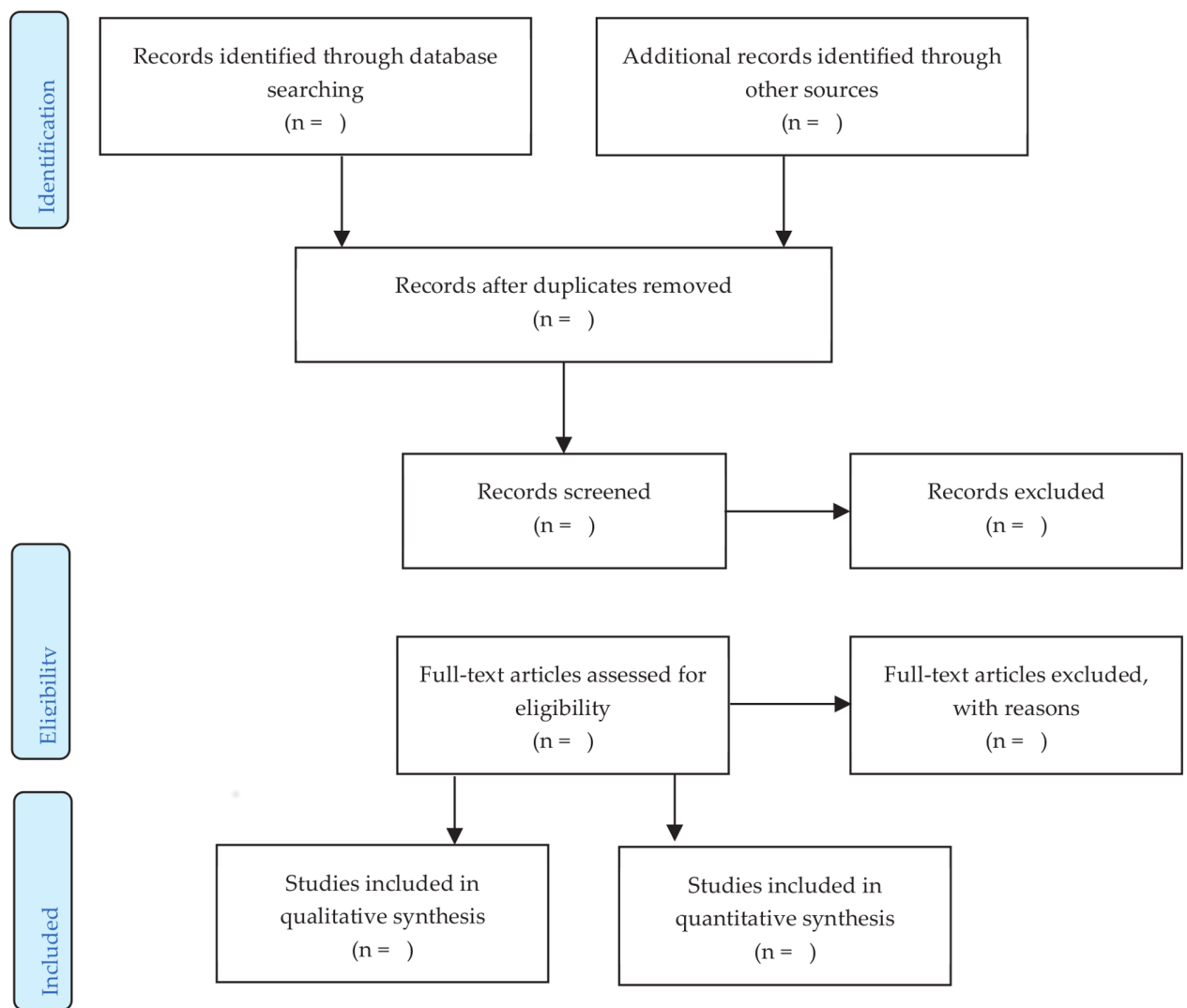

Figure 1 Overview study selection process.

study design checklists from the Critical Appraisal Skills Programme (CASP).${ }^{27}$ As, CASP lack a checklist for mixed methods studies, the mixed method appraisal tool will be applied..$^{28}$ In the case of any ambiguity concerning a study's quality assessment, an additional reviewer is going to be consulted. No exclusion of eligible studies will be made on behalf of the quality assessment as studies with limited quality nevertheless can provide a valid rationale as guidance as to where more research is required.

\section{Stage 4: charting data}

A data charting form would be developed, and piloted on the first 5 to 10 included studies in this review. The piloting will support the team to reach an agreement on extraction consistency. The latter is especially important, as the extraction will be conducted individually and independently by a minimum of two reviewers. ${ }^{17}$ A systematic and analytical approach will be utilised to extract the relevant information of each included study. The variables and themes to be included in order to answer the review's objective and questions to the literature will be established iteratively (box 1). Thus, the data charting form will be updated throughout the review by one of the reviewers (FW) who will also hold regular discussion with the others in the review team. ${ }^{17}$

Influenced by Weingarten, Paul and Leibovici's substantial contribution to raise the ethical awareness in reviews, an ethical assess form (box 2) was developed for the upcoming scoping review including five requirements. ${ }^{29}$ Included studies valued by the review team as not adhering to the ethical requirements will be excluded at this stage of the scoping review process.

\section{Stage 5: collating, summarising and reporting the results}

In the fifth stage, an overview and narrative account of variables and information extracted in stage 4 will be presented, and as highlighted by Arksey and O'Malley no evidence grading will be executed. ${ }^{16}$ Levac, Colquhoun

\section{Box 1 Tentative data charting form}

Author and date.

Study title.

Journal full reference.

Aim, objective and/or research questions.

Study and recruitment context (eg, in what country and where people were recruited).

Participant characteristics (eg, age, gender, education year/semester of study, course (ie, theoretical or clinical placement)).

Sampling method.

Number of study participants.

Study design.

Data collection (eg, what data collection methods were used?).

Data analysis (eg, how was the data analysed?).

Described ethical approval and/or considerations. ${ }^{29}$

Described teaching strategies and/or interventions targeting review focus.

Described outcomes and assessments.

Most relevant findings.

Study quality appraisal. ${ }^{27} 28$ 
Box 2 Tentative ethical requirements influenced by Weingarten, Paul and Leibovici.

Was the study approved by a research ethical committee? (Yes/No)

- Was informed consent obtained? (Yes/No)

- Were adequate measurements taken to protect personal data? (Yes/ No)

- Is there a declaration on financial support? (Yes/No)

- Is there a declaration on potential conflict of interest? (Yes/No)

and O'Brien ${ }^{17}$ and Daudt, Van Mossel and Scott ${ }^{18}$ suggest that the extracted qualitative data should be presented through thematic analysis, since no synthesis of data is required. ${ }^{16}$ For this purpose, the thematic analysis by Braun and Clarke will be applied which is a flexible method suitable when the data is broad and allowing for a wide range of analytical options. ${ }^{30}$ This cohere with the upcoming scoping review, which will include studies with a wide range of research questions and methods. Quantitative data will be reviewed through basic descriptive numerical analysis and presented in tables and charts to highlight the range of data. ${ }^{16}$ If studies with a mixed method design are included in stage 3 , the qualitative and quantitative data will be extracted and analysed separately. A minimum of two reviewers will be responsible for this stage of the scoping review process. During the process, meetings with the entire review team will be scheduled by the first reviewer (FW) to discuss and come to agreement concerning analysis and presentation of extracted data.

\section{Stage 6: consultation stage}

To validate the findings of this scoping study and make it more useful for practice the optional stage consultation will be applied. For this purpose, the findings from the scoping review will be presented to a group of educators and students connected to a nursing programme as a means to contribute with valuable insights on issues connected to the application and implementation of the findings.

\section{Patient and public involvement}

No patients have been involved in the design of this study. However, to conduct a study targeting teaching strategies for critical thinking in nursing education will eventually benefit patients since education is the foundation for raising future nurses and improve patient care.

\section{ETHICS AND DISSEMINATION}

Under the Swedish Ethical Review Act $(2003: 460)^{31}$ this study does not need ethical clearance by a Regional Ethical Review Authority as it does not include any primary empirical data on biological material or sensitive information (eg, ethnicity, political or sexual orientation). However, the issue of ethical consideration in the execution of reviews is raised by Vergnes $e t a \vec{l}^{2}$ as well as by Weingarten, Paul and Leibovici. ${ }^{29}$ They state that without an ethical judgement of the included studies it could result in establishing clinical practise and guidelines based on studies with poor ethical quality and even unethical studies. It could further be seen as a way of increasing the awareness and necessity of high ethical standards in research. To meet these requirements one variable in the charting form will be ethical consideration and for that purpose a tentative checklist for ethical requirements was developed (box 2). The checklist will be tested on a minimum of 10 publications, and revised accordingly if necessary.

The upcoming scoping review will contribute to the advancement of research concerning teaching strategies targeting nursing students' skills and abilities in critical thinking and the outcome assessment of it. It will also provide an indication of the maturity of the literature by identifying research gaps. Gaining more knowledge of the targeted research area can act as a benchmark to implement new teaching strategies facilitating students' critical thinking disposition within the nursing education. This will better prepare future nurses for the complex care situations they will approach. Our findings will be used to inform the design of a future study aiming to develop and evaluate an educational intervention targeting teaching strategies focussing on nursing students' critical thinking skills and abilities. The upcoming scoping review will be published in a peer-reviewed journal. We expect to report in late spring 2020.

Contributors FW, EC, AW and GB were responsible for the initial design of this study. FW conceptualised the review approach and led the writing of the manuscript. FW, EC, AW and GB contributed to the protocol's development and approved the final version of this protocol. GB, EC and AW led the supervision of the manuscript preparation.

Funding The authors have not declared a specific grant for this research from any funding agency in the public, commercial or not-for-profit sectors.

Competing interests None declared.

Patient consent for publication Not required.

Provenance and peer review Not commissioned; externally peer reviewed.

Open access This is an open access article distributed in accordance with the Creative Commons Attribution Non Commercial (CC BY-NC 4.0) license, which permits others to distribute, remix, adapt, build upon this work non-commercially, and license their derivative works on different terms, provided the original work is properly cited, appropriate credit is given, any changes made indicated, and the use is non-commercial. See: http://creativecommons.org/licenses/by-nc/4.0/.

ORCID iD

Frida Westerdahl http://orcid.org/0000-0002-4086-0086

\section{REFERENCES}

1 Von Colln-Appling C, Giuliano D. A concept analysis of critical thinking: a guide for nurse educators. Nurse Educ Today 2017;49:106-9.

2 Cronenwett L, Sherwood G, Barnsteiner J, et al. Quality and safety education for nurses. Nurs Outlook 2007;55:122-31.

3 Chang MJ, Chang Y-J, Kuo S-H, et al. Relationships between critical thinking ability and nursing competence in clinical nurses. $J$ Clin Nurs 2011;20:3224-32.

4 Andrea Sullivan E, Sullivan EA. Critical thinking in clinical nurse education: application of Paul's model of critical thinking. Nurse Educ Pract 2012;12:322-7.

5 Scheffer BK, Rubenfeld MG. A consensus statement on critical thinking in nursing. J Nurs Educ 2000;39:352-9.

6 Chan ZCY. A systematic review of critical thinking in nursing education. Nurse Educ Today 2013;33:236-40. 
7 Alfaro-LeFevre R. Critical thinking, clinical Reasoning, and clinical judgement a practical approach. 6th edn. Philadelphia, PA: Elsevier, 2017.

8 Victor-Chmil J. Critical thinking versus clinical Reasoning versus clinical judgment: differential diagnosis. Nurse Educ 2013;38:34-6.

9 Manetti W. Sound clinical judgment in nursing: a concept analysis. Nurs Forum 2019;54:102-10.

10 Burrell LA. Integrating critical thinking strategies into nursing curricula. Teach Learn Nurs 2014;9:53-8.

11 Simpson E, Courtney M. Critical thinking in nursing education: literature review. Int J Nurs Pract 2002;8:89-98.

12 Carvalho DPSRP, Azevedo IC, Cruz GKP, et al. Strategies used for the promotion of critical thinking in nursing undergraduate education: a systematic review. Nurse Educ Today 2017;57:103-7.

13 De Oliviera LB, Díaz LJR, Carbogim FDC, et al. Effectiveness of teaching strategies on the development of critical thinking in undergraduate nursing students: a meta-analysis. Rec Esc Enferm USP 2016;50:350-9.

14 Zuriguel Pérez E, Lluch Canut MT, Falcó Pegueroles A, et al. Critical thinking in nursing: Scoping review of the literature. Int $J$ Nurs Pract 2015;21:820-30.

15 Carter AG, Creedy DK, Sidebotham M. Efficacy of teaching methods used to develop critical thinking in nursing and midwifery undergraduate students: a systematic review of the literature. Nurse Educ Today 2016;40:209-18.

16 Arksey H, O'Malley L. Scoping studies: towards a methodological framework. Int J Soc Res Methodol 2005;8:19-32.

17 Levac D, Colquhoun H, O'Brien KK. Scoping studies: advancing the methodology. Implement Sci 2010;5:69.

18 Daudt HML, van Mossel C, Scott SJ. Enhancing the scoping study methodology: a large, inter-professional team's experience with Arksey and O'Malley's framework. BMC Med Res Methodol 2013;13:48.
19 Tricco AC, Lillie E, Zarin W, et al. PRISMA extension for scoping reviews (PRISMA-ScR): checklist and explanation. Ann Intern Med 2018;169:467-73.

20 Colquhoun HL, Levac D, O'Brien KK, et al. Scoping reviews: time for clarity in definition, methods, and reporting. J Clin Epidemiol 2014;67:1291-4.

21 Mheta D, Mashamba-Thompson TP. Barriers and facilitators of access to maternal services for women with disabilities: Scoping review protocol. Syst Rev 2017;6:99.

22 Banning M. Approaches to teaching: current opinions and related research. Nurse Educ Today 2005;25:502-8.

23 Stoffels M, Peerdeman SM, Daelmans HEM, et al. Protocol for a scoping review on the conceptualisation of learning in undergraduate clinical nursing practice. BMJ Open 2019;9:e024360.

24 Ouzzani M, Hammady H, Fedorowicz Z, et al. Rayyan-a web and mobile APP for systematic reviews. Syst Rev 2016;5:210.

25 Moher D, Liberati A, Tetzlaff J, et al. Preferred reporting items for systematic reviews and meta-analyses: the PRISMA statement. PLoS Med 2009;6:e1000097.

26 Grant MJ, Booth A. A typology of reviews: an analysis of 14 review types and associated methodologies. Health Info Libr J 2009;26:91-108.

27 CASP. Critical appraisal skills programme, 2018. Available: https:// casp-uk.net/casp-tools-checklists/ [Accessed 26 Jul 2019].

28 Hong QN, Pluye P, Fbregues S, et al. Mixed methods appraisal tool (MMAT), version 2018. Registration of Copyright (\#1148552), Canadian Intellectual Property Office, Industry Canada.

29 Weingarten MA, Paul M, Leibovici L. Assessing ethics of trials in systematic reviews. BMJ 2004;328:1013-4.

30 Braun V, Clarke V. Using thematic analysis in psychology. Qual Res Psychol 2006;3:77-101.

31 Act concerning the ethical review of research involving humans (SFS 2003:460).

32 Vergnes J-N, Marchal-Sixou C, Nabet C, et al. Ethics in systematic reviews. J Med Ethics 2010;36:771-4. 